\title{
Application of GIS in downscaling regional climate model results over the province of Ontario
}

\author{
Yang Zhou ${ }^{1,2^{*}}$ and Jinyu Zhang ${ }^{2}$
}

\begin{abstract}
Background: Climate change is a significant and long-term change in the weather patterns over periods ranging from decades to millions of years. The impacts of climate change have been drawn more and more worldwide attention. To study the impacts, general circulation models (GCMs) were developed to simulate climate change at a global scale. The climate information obtained from GCMs is usually at a fairly coarse resolution. In comparison, regional climate models (RCMs) work in a small area of interest and can provide climate information at resolution as fine as $25-50 \mathrm{~km}$. When higher resolution climate information is needed and the applied RCMs are incapable of undertaking the task, statistical downscaling techniques can be introduced to acquire the desired climate information. In this study, three interpolation methods are applied to downscale regional climate model (RCM) results for higher resolution climate information at $10 \mathrm{~km}$.

Results: The results indicated that the three interpolation methods could generate high-quality estimates at $10 \mathrm{~km}$ grids. The downscaled RCM results approximated to the $10 \mathrm{~km}$ official data which was published by Agriculture and Agri-Food Canada, Government of Canada. Compared with the results of IDW and spline methods, the results obtained from kriging method generated smoother interpolation map and showed modest variations in the difference map.
\end{abstract}

Conclusions: All the three interpolation methods could fulfill the task of downscaling the RCM results from $25 \mathrm{~km}$ to $10 \mathrm{~km}$. Overall, kriging interpolation method showed better performance than the other two methods in this study.

Keywords: Regional climate model; Interpolation; Downscaling; ArgGIS

\section{Background}

Climate change is a significant and long-term change in the weather patterns over periods ranging from decades to millions of years (Gabri 2012). Around the world, landscape is changing, temperature is rising and season is shifting. The impacts of climate change are drawing more and more worldwide attention. Across Canada, all the provinces and territories are experiencing the effects of a changing climate, though the specific impacts vary by region (Lemmen et al. 2008). In order to enhance knowledge and scientific expertise on the effects of climate change in the province of Ontario, Ontario Ministry of the Environment sponsored a project titled "Regional Climate

\footnotetext{
* Correspondence: zhou206y@uregina.ca
${ }^{1}$ Institute for Energy, Environment and Sustainable Communities, University of

* Correspondence: zhou206y@uregina.ca
${ }^{1}$ Institute for Energy, Environment and Sustainable Communities, University of Regina, Regina, Saskatchewan S4S 0A2, Canada

${ }^{2}$ Faculty of Engineering and Applied Science, University of Regina, Regina, Saskatchewan S4S 0A2, Canada
}

\section{Springer}

Modelling over Ontario using UK PRECIS" to study climate change in this region (Center for Studies in Energy and Environment 2011). PRECIS (Providing Regional Climates for Impacts Studies) is a well-established regional climate modelling system that can help to provide high resolution climate information for impacts studies worldwide. Different from General Circulation Models (GCMs), which simulate the change in climate at a global scale and provide a fairly coarse horizontal resolution (usually over $100 \mathrm{~km}$ ) in climate information, the Regional Climate Models (RCMs) work in a small, limited area of interest and can give climate information at resolution as fine as $25-50 \mathrm{~km}$ (Jones et al. 2004). In this project, the climate information outputted from the PRECIS regional climate model was at a resolution of $25 \mathrm{~km}$.

Over the past decades, the application of interpolation techniques had drawn more attention in climate analysis, and GIS-based spatial interpolation methods had been 


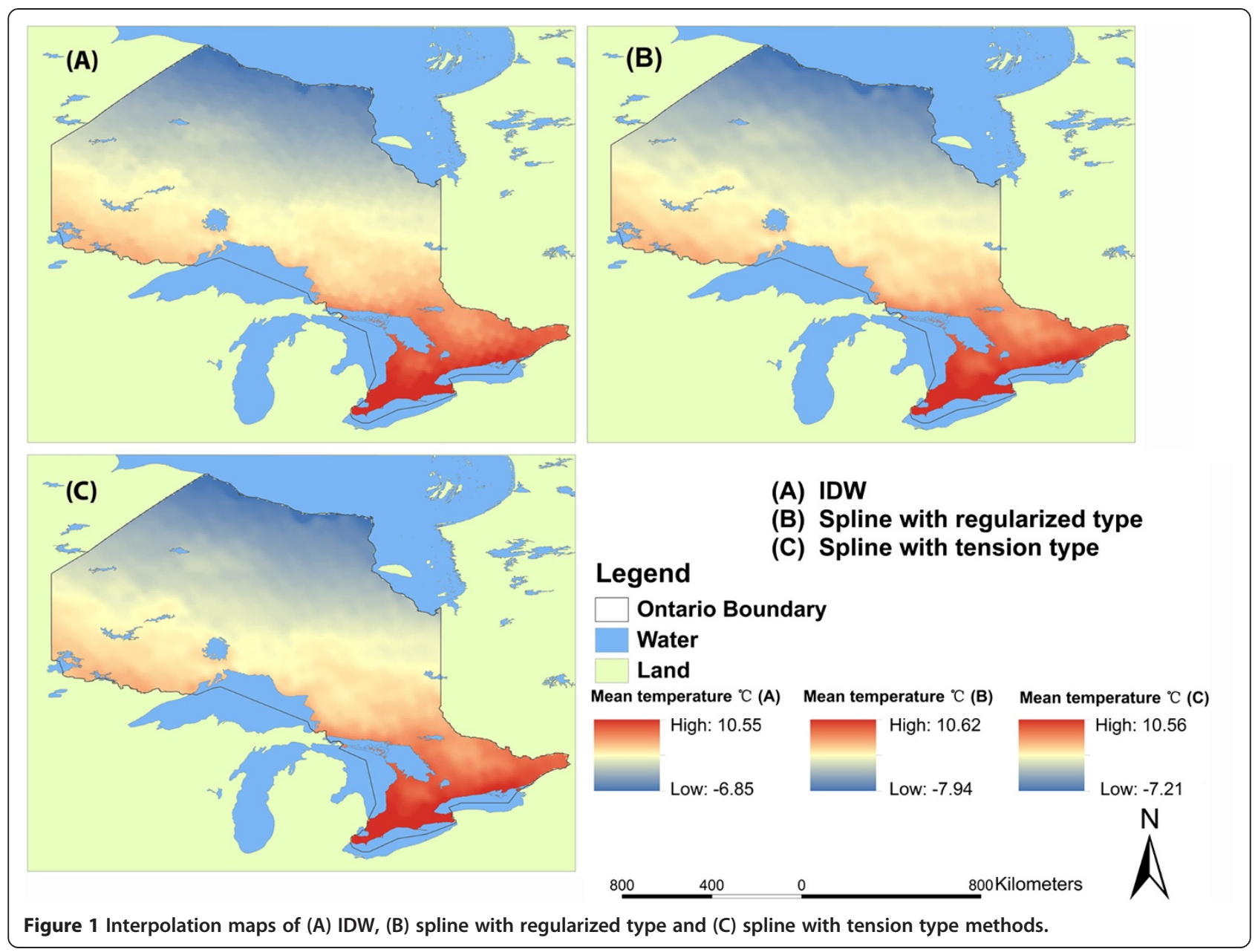

widely employed for climate analysis (Jeffrey et al. 2001; Naoum and Tsanis 2004; Hijmans et al. 2005; Hong et al. 2005; Van der Heijden and Haberlandt 2010; Wu and Li 2013). Naoum and Tsanis (2004) introduced several common interpolation methods in GIS and compared the performance of each method in a case study of rain gauges in Switzerland. They concluded that the spline_tension, IDW, and kriging methods could be able to provide reliable results. Hijmans et al. (2005) applied a spatial interpolation technique to obtain a very high resolution climate surface for global land areas. They applied the thin-plate smoothing spline algorithm for interpolation. In order to improve the overall accuracy of results, the authors suggested spatial interpolation could be carried out at a regional scale. Hong et al. (2005) employed spline interpolation techniques to develop a gridded climate database for China. They concluded that the thin-plate soothing spline interpolation method was better than other methods, especially, in computation efficiency and accuracy. Van der Heijden and Haberlandt (2010) employed four interpolation techniques, which are nearest neighbor $(\mathrm{NN})$, inverse distance weighting (IDW), ordinary kriging (OK) and kriging with external drift (KED), to figure out which interpolation method would have strong influence on daily discharge and monthly nitrate simulation within a SWAT model. They concluded that kriging with external drift interpolation method generally outperformed the other interpolation methods in their case study. Overall, the past studies indicate interpolation techniques are feasible and effective ways for climate analysis. However, there is no specific technique suitable for all cases. The best way is to apply different techniques in the same study and to see which one outperforms others.

This study is based on the project "Regional Climate Modelling over Ontario using UK PRECIS". In the project climate information was provided at a resolution of $25 \mathrm{~km}$. However, when higher resolution climate information such as $10 \mathrm{~km}$ climate information is demanded in researches, the applied PRECIS regional climate model may become incapable for the demand. Statistical interpolation techniques, therefore, can be introduced to fulfill the task. The higher resolution climate information can be obtained through downscaling the previous RCM results. 
In this study, three interpolation methods, inverse distance weighting (IDW), spline, and kriging, are employed to downscale the results of the project from $25 \mathrm{~km}$ to $10 \mathrm{~km}$. There are two major purposes for this study: (1) verifying the applicability of interpolation techniques in downscaling the results of regional climate model and (2) identifying which technique provided in ArcGis outperforms others. The $25 \mathrm{~km}$ RCM results obtained from the baseline of the project are interpolated via the three interpolation methods in ArcGIS. To assess the performance of the three interpolation methods in downscaling the RCM results, the $10 \mathrm{~km}$ official data provided by Agriculture and Agri-Food Canada, Government of Canada, are collected for further comparison.

\section{Results and discussion}

The three interpolation methods were employed respectively to generate the 25 years annual mean temperature map over the province of Ontario. Two types of spline interpolation method - regularized type and tension type were applied in this study. In kriging methods, we applied ordinary kriging (OK) method. Three ordinary kriging models, spherical, exponential and linear model, were assessed respectively. In Figure 1, it showed the 25 years annual mean temperature interpolation map generated by IDW and the two types of spline method. The interpolation map of IDW method was shown in the map A of Figure 1. From this map, we obtained a general temperature trend in the province of Ontario: the north of Ontario had the lowest annual mean temperature while the south had the highest. This corresponded to the common sense that the temperature usually decreases as the latitude increased in the Northern Hemisphere. Same conclusion can also be obtained from the maps B and C in Figure 1. Compared the interpolation map of IDW method with that of spline method, we could see that spline method generated much smoother map than IDW method; however, there was no apparent difference between the maps of the two types of spline methods. The Figure 2 compared the interpolation maps of the three models in ordinary kriging method. There was no visually detectable difference among the three interpolation surfaces. Next, the comparison among the three interpolation methods was given in Figure 3. Compared with the IDW and spline methods, the ordinary kriging method could

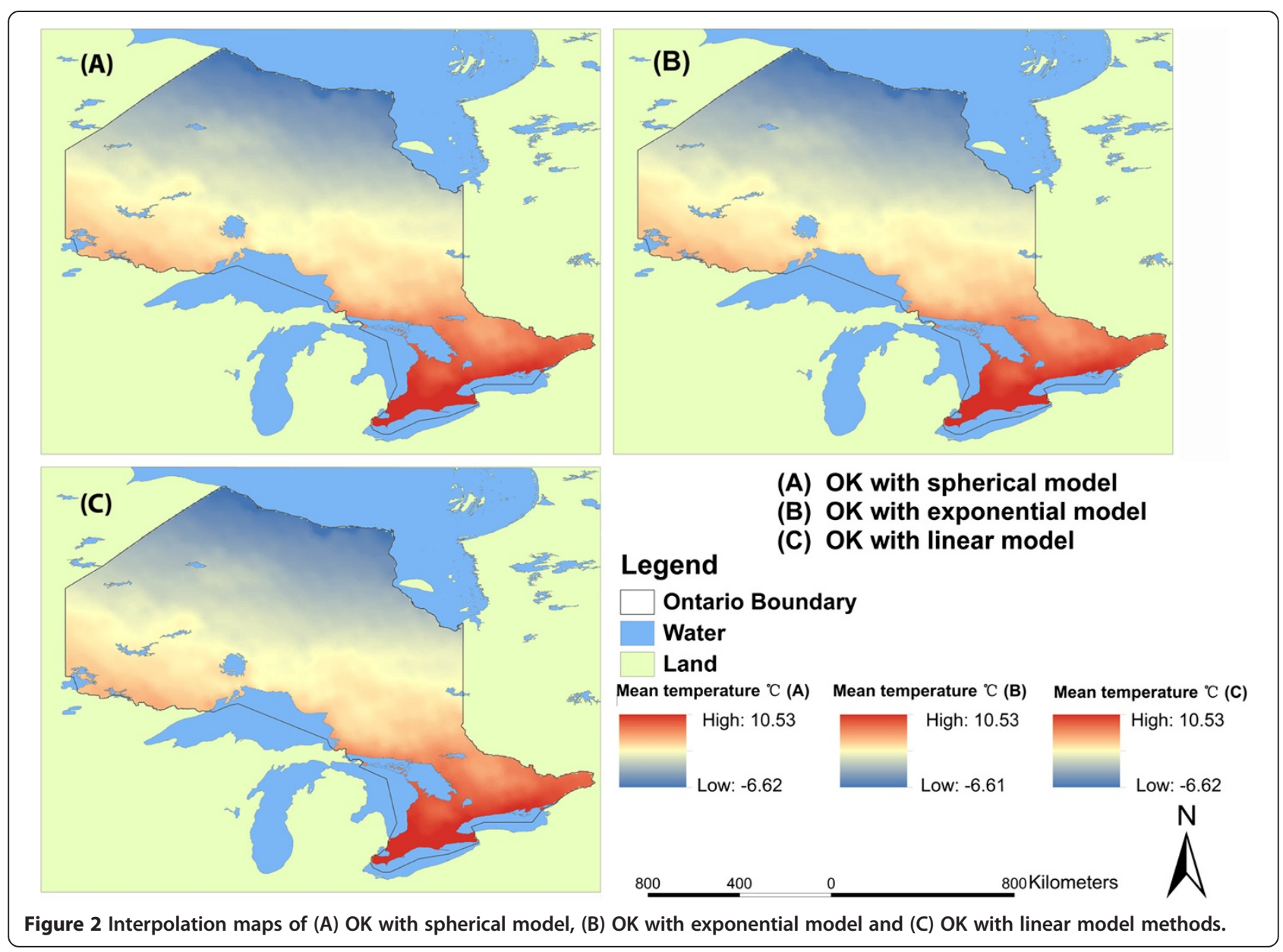




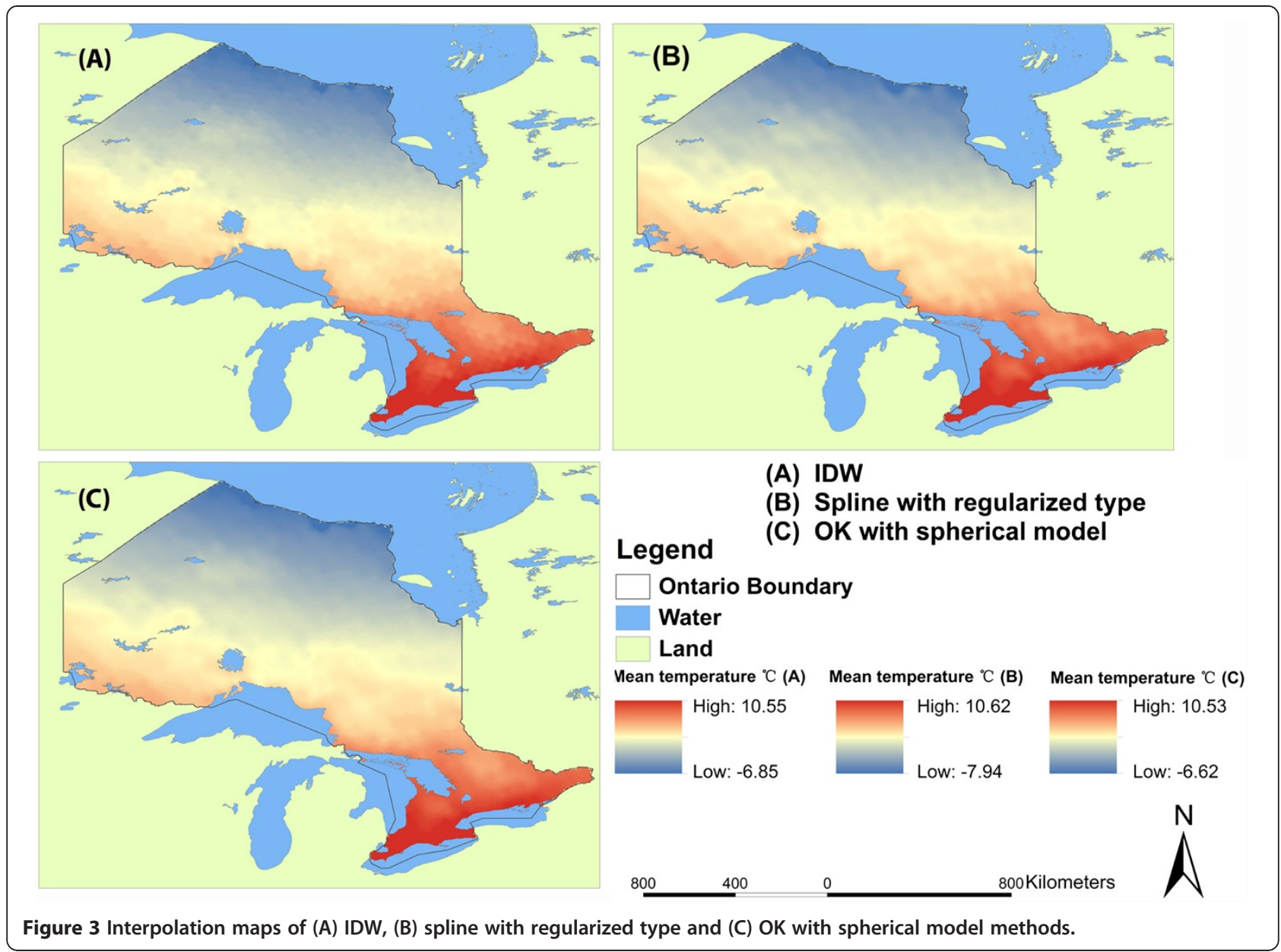

generate the smoothest interpolation map. In this map, the highest annual mean temperature is 10.53 degree Celsius while the lowest is -6.62 degree Celsius. There was no much difference in the highest mean temperature among the three maps; however, the lowest mean temperature from the spline method was lower than the other two methods more than one degree Celsius. Table 1 summarized the results of the six applied interpolation approaches. We could see that all the six approaches' interpolation results were higher than the official
$10 \mathrm{~km}$ data. Their mean values were approximately 0.64 degree Celsius higher than the official data. Interestingly, their standard deviations were quite same.

In order to assess the downscaled RCM results in terms of how well the interpolated estimates fit the $10 \mathrm{~km}$ official data, the interpolated estimates were extracted from the interpolation map based on the coordinates of $10 \mathrm{~km}$ official data. After the interpolated estimates were obtained, a statistical software named Minitab was employed for further analysis. The Figure 4 showed the

Table 1 Summary of the six applied approaches

\begin{tabular}{lllll}
\hline Methods & Minimum ${ }^{\circ} \mathbf{C}$ & Maximum ${ }^{\circ} \mathbf{C}$ & Mean ${ }^{\circ} \mathbf{C}$ & Standard deviation ${ }^{\circ} \mathbf{C}$ \\
\hline IDW & -6.85 & 10.55 & 1.96 & 3.12 \\
Spline method with regularized type & -7.94 & 10.62 & 1.96 & 3.13 \\
Spline method with tension type & -7.21 & 10.56 & 1.96 & 3.13 \\
OK with spherical model & -6.62 & 10.53 & 1.96 & 3.13 \\
OK with exponential model & -6.61 & 10.53 & 1.96 & 3.13 \\
OK with linear model & -6.62 & 10.53 & 1.96 & 3.13 \\
Official 10 km data & -4.56 & 9.90 & 1.32 & 3.13 \\
\hline
\end{tabular}



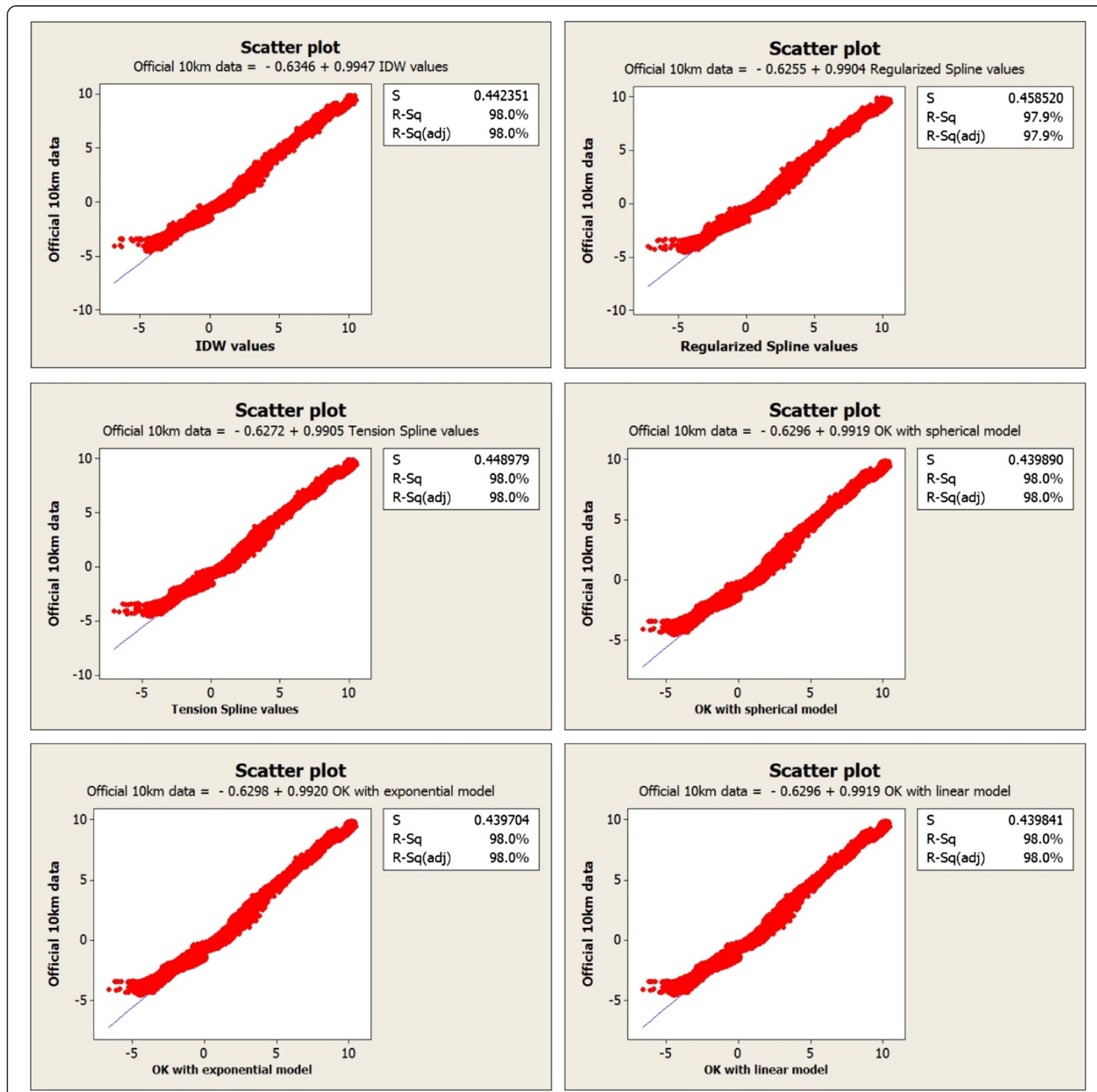

Figure 4 Scatter plots of the six applied approaches.

scatter plots of the six approaches. We could see that although the left tail was slightly away from the 45 degree line, most of the points fell around the 45 degree line indicating the interpolated estimates well approximated to the $10 \mathrm{~km}$ official data. In addition, the statistic index, R-Sq, pointed out how well the interpolated estimates fit the official data and the higher the R-Sq was, the more fit the estimates would be. In this study, all the R-Sq values from the six approaches were $98 \%$ except spline method with regularized type; but it was merely $0.1 \%$ lower than the other approaches. Accordingly, all the six interpolation methods could generate well estimates at the $10 \mathrm{~km}$ official data's grids. Consequently, we concluded that all the three interpolation methods (IDW, spline and kriging) could be qualified to fulfill the task of downscaling RCM results for higher resolution climate information in regional scale.

Furthermore, in order to examine the difference between the interpolated RCM estimates and the $10 \mathrm{~km}$ official data, the dataset that contains the difference was constituted by subtracting the values of the official data from the values of the interpolated estimates. 
The difference map was shown in Figure 5. The red color indicated the interpolated estimates were overestimated, while the blue color indicated the interpolated estimates were underestimated. The light green area implied the difference between the interpolated estimates and the official data was insignificant in this area. From Figure 5, we could see that the interpolated estimates were overestimated in the most areas of the middle Ontario and the interpolated RCM estimates well approximated to the $10 \mathrm{~km}$ official data in the Northeast and the partial south areas of Ontario. Only in several small areas of the north Ontario, the interpolated estimates were underestimated. The difference maps for the three interpolation methods were given in Figure 6. Compared with the difference maps of IDW and spline methods, the difference map for kriging method showed relatively modest variations in color. This implied the interpolated estimates from kriging method had the smallest difference with the $10 \mathrm{~km}$ official data.

\section{Conclusions}

Three interpolation methods, IDW, spline and kriging, were employed in this study to assess their performance for RCM downscaling. Compared with IDW and spline methods, the kriging method could create smoother interpolation map. After their interpolation maps were obtained, the RCM interpolated estimates at official $10 \mathrm{~km}$ grids were extracted from the interpolation maps based on the coordinates of the official data. The interpolated estimates were further analyzed via a statistics software. The results showed that the 25 years annual mean temperatures obtained from the three interpolation methods were around 0.64 degree Celsius higher than the official data, indicating general overestimation in the interpolated results. However, the overestimation did not significantly influence the results of interpolated estimates since the statistic index, R-Sq, indicated the interpolated estimates well approximated to the $10 \mathrm{~km}$ official data. In order to assess the difference between the interpolated estimates and the $10 \mathrm{~km}$ official data, the difference maps over the province of Ontario were generated. In most area of middle Ontario the interpolated estimates were overestimated while the differences were small in the Northeast and partial south Ontario. Compared the three difference maps obtained from the three interpolation methods, the map of kriging method showed modest variations in color. Therefore, the interpolated estimates from kriging method were closer to the official data. In conclusion, all the three interpolation methods in GIS could fulfill the task of

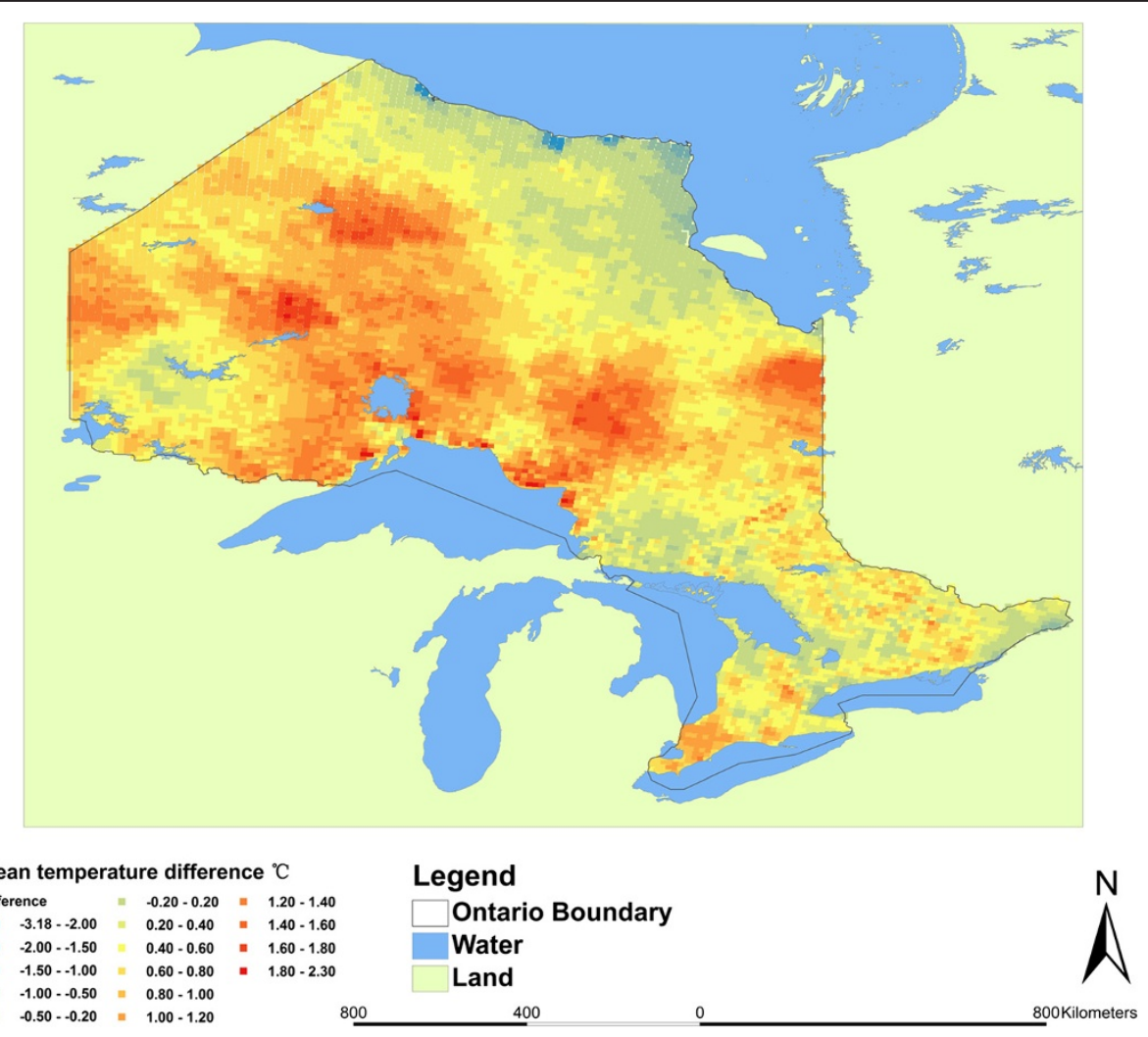

Figure 5 Difference map of the IDW interpolation method. 


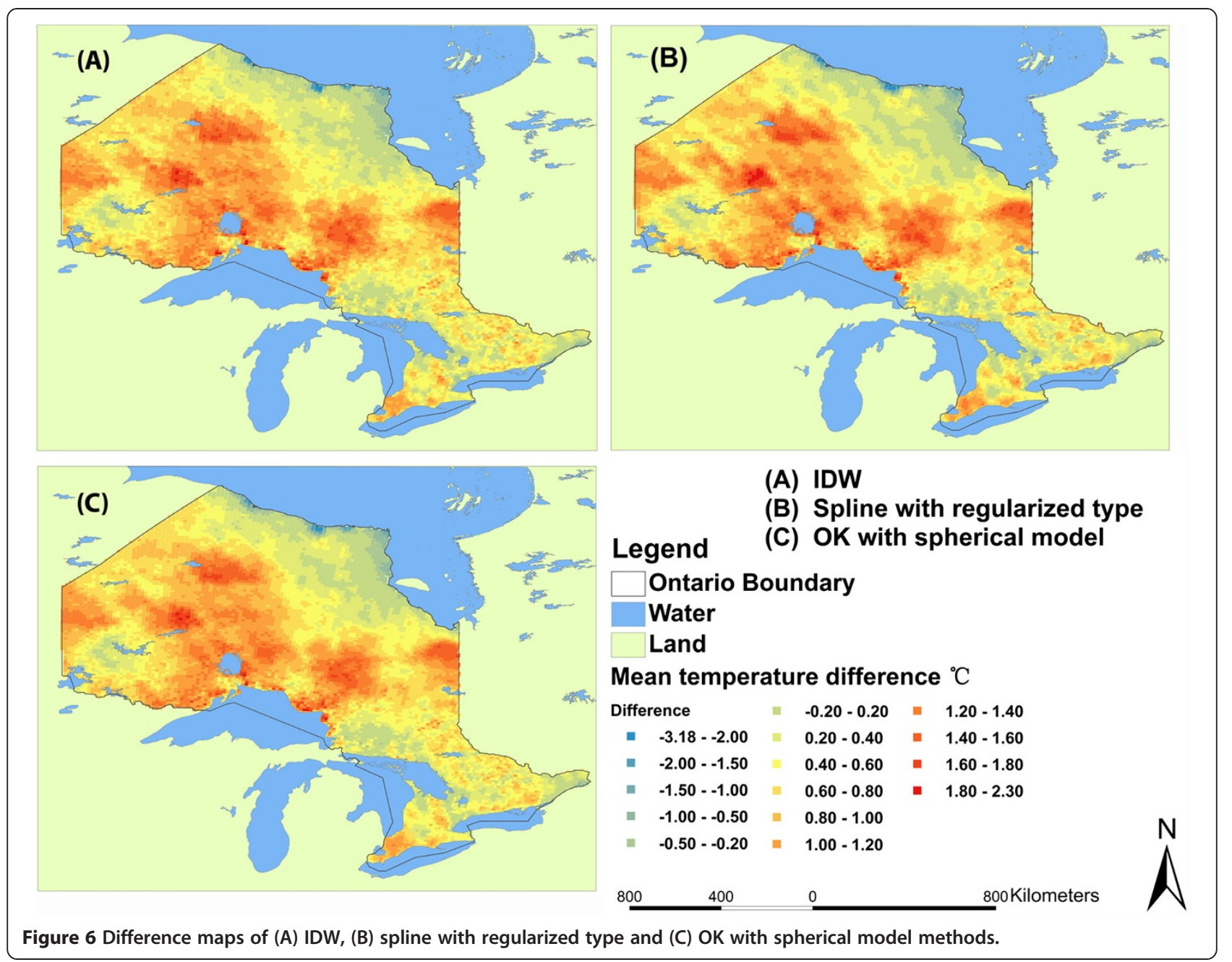

downscaling the RCM results from $25 \mathrm{~km}$ to $10 \mathrm{~km}$. The downscaled RCM estimates approximated to the official $10 \mathrm{~km}$ data. In this study, the kriging method showed better performance than the other two methods. It generated smoother interpolation map and had modest variations in the difference map.

\section{Methods}

\section{Study area}

Ontario is Canada's most populous province and second largest province in total area. It is home to the nation's most populous city, Toronto and the nation's capital, Ottawa. The total area of Ontario is $1.1 \times 106 \mathrm{~km}^{2}$, of which $85.3 \%$ is land and $14.7 \%$ is water (http://en.wikipedia.org/ wiki/Ontario). Two-thirds of Ontario is covered by the Canadian Shield, which contains Precambrian rocks that are over 570 million years old. The rest Ontario in the north are the Hudson Bay Lowlands, consisting of swamp, meadow and forest. The landscape of Ontario is characterized by forests. Sixty-six per cent of Ontario is classified as forested land, totaling $0.7 \times 106 \mathrm{~km}^{2}$, which accounts for about $2 \%$ of the world's forests. (http://www.ontario.ca/government/about-ontario). The climate in Ontario is humid continental in the south and subarctic in the north. The large bodies of water within the province have a moderating effect on climate, making summer and winter temperatures less extreme and the difference between day and night temperatures smaller. During the past decades, climate change has brought many impacts upon human societies around the world, including Ontario. The Ontario government has conducted many researches and programs to help people adapt to the effects that are already being experienced, as well as the future effects of climate change (Baldwin et al. 2000).

\section{Data collection \& project procedures}

Two sets of datasets were employed in this study. The primary dataset, $25 \mathrm{~km}$ regional climate modeling (RCM) data over the province of Ontario, was obtained from the 
website (http://env.uregina.ca/moe/). This RCM result dataset over the province of Ontario was generated by a well-established Providing Regional Climates for Impacts Studies (PRECIS) model. In this study, the annual mean temperature would be taken into consideration and this dataset would be processed as the source dataset that to be interpolated through the three interpolation methods in ArcGIS.

The secondary dataset, daily $10 \mathrm{~km}$ gridded climate dataset for Canada 1961-2003, was obtained from the website (www.agr.gc.ca/nlwis-snite/index_e.cfm). It was provided by Agriculture and Agri-Food Canada, Government of Canada. The Daily $10 \mathrm{~km}$ Gridded Climate dataset for Canada south of $60^{\circ}$ north contained the coordinates of locations as well as the associated daily maximum temperature, minimum temperature, and precipitation data. In this dataset, the desired climate information for $10 \mathrm{~km}$ was stored in a number of individual files and the climate information was offered by daily data. Therefore, pretreatments of this dataset was necessary for obtaining the climate data that contained annual temperature information in one file. The desired $10 \mathrm{~km}$ annual mean temperature dataset could be obtained based on the data of maximum and minimum temperature.

The primary dataset was based on the period of 1979 to 2009 while the secondary dataset was based on the period of 1961 to 2003. Therefore, we considered the period of 1979 to 2003 as our study period and the 25 years mean temperature would be studied. The flowchart of this project was given in Figure 7. It showed the detailed procedures of this study.

\section{Spatial interpolation methods in a GIS}

The following is a brief introduction of the interpolation methods available in ArcGis desktop 10.

\section{Inverse Distance Weighting (IDW)}

Inverse distance weighting (IDW) is an interpolation method that interpolated estimates are made based on values at nearby locations weighted by distance from the interpolation location. IDW works under a basic assumption that nearby points ought to be more closely related to the value of interpolated location than distant points (Naoum and Tsanis 2004). In IDW method, estimation at interpolated location is a weighted linear combination, with the weights $\lambda_{1}$ being inversely proportional to the square distance between sampled location $\mu_{1}$ and the point of estimate $\mu$ (Van der Heijden and Haberlandt 2010):

$$
Z_{I D W}(\mu)=\frac{1}{\sum_{i=1}^{n(\mu)} \lambda_{i}(\mu)} \sum_{i=1}^{n(\mu)} \lambda_{i}(\mu) Z\left(\mu_{i}\right)
$$

with

$$
\lambda_{i}(\mu)=\frac{1}{\left|\mu-\mu_{i}\right|}
$$

\section{Spline}

Spline interpolation consist of the approximation of a function by means of series of polynomials over

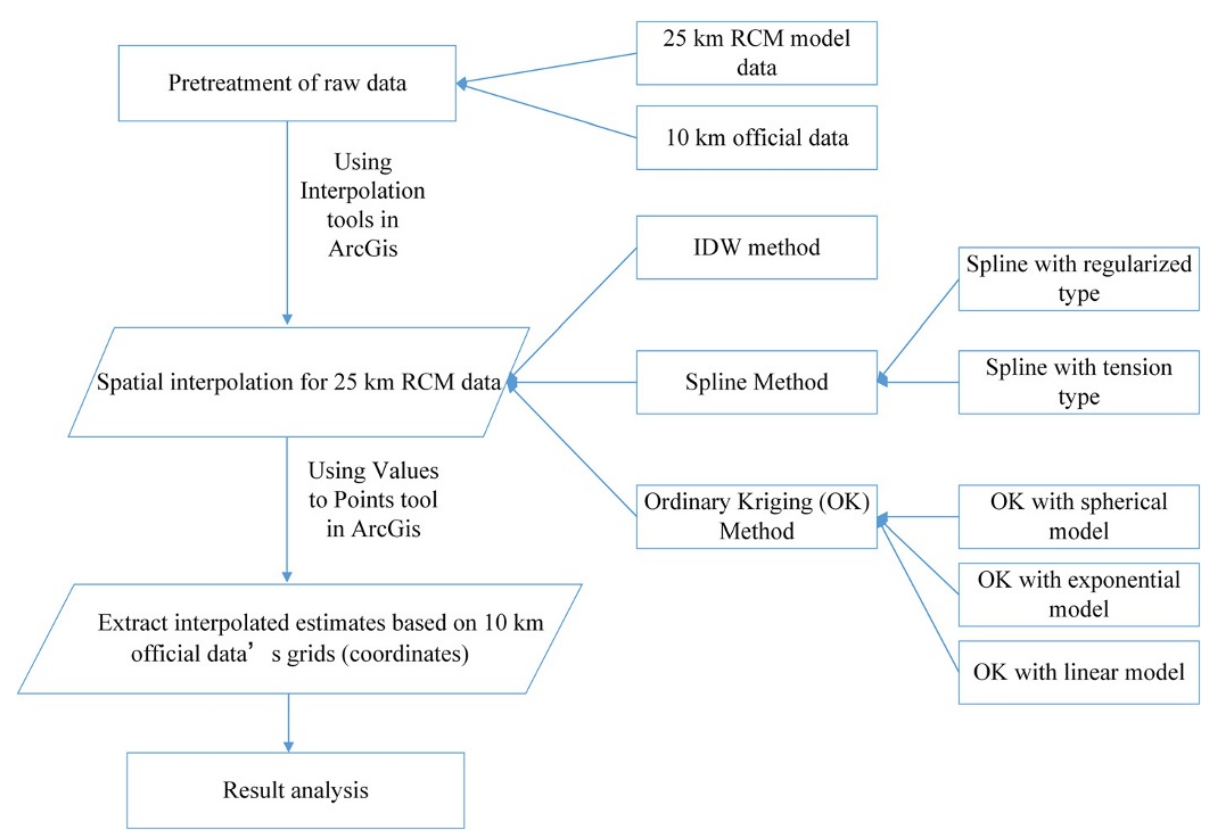

Figure 7 Flowchart of the study. 
nearby intervals with continuous derivatives at the end-point of the intervals (Naoum and Tsanis 2004). Smoothing spline interpolation enables the variance of the residuals over the dataset being controlled. The interpolation estimates are calculated by an iterative algorithm. In spline interpolation, the interpolated surface must precisely pass through the data points; meanwhile, the surface must have minimum curvature (Naoum and Tsanis 2004). ArcGIS provides two types of spine interpolation methods which are regularized type and tension type.

\section{Kriging}

Kriging is based on regionalized variable theory that provides optimal interpolation estimates at given coordinate locations. It involves an interactive investigation into the spatial behavior of the phenomenon before outputting the interpolation surface. In the regionalized variable theory, it assumes that the spatial variation is statistically homogeneous through the surface, which means the same pattern of variation can be captured at all locations on the surface. The hypothesis of spatial homogeneity is the basis of the regionalized variable theory (Naoum and Tsanis 2004).

\section{Competing interests}

The authors declared that they have no competing interest.

\section{Authors' contributions}

YZ conceived of this study, and carried out data pretreatment, results analysis, and manuscript drafting. JZ carried out data collection and participated in drafting the manuscript. All authors read and approved the final manuscript.

Received: 11 December 2013 Accepted: 21 January 2014

Published: 30 January 2014

\section{References}

Baldwin DJB, Desloges JR, Band LE (2000) Physical Geography of Ontario. In: Perera AH, Euler DE, Thompson I (eds) Ecology of a Managed Terrestrial Landscape: Patterns and Processes of Forest Landscapes in Ontario. University of British Columbia Press, Vancouver, British Columbia, Canada, pp 12-299

Center for Studies in Energy and Environment (2011) Regional Climate Modelling over Ontario using UK PRECIS., http://env.uregina.ca/moe/

Gabri J (2012) Global Climate Change., http:/hetf.org/index.php/global-climate-change

Hijmans RJ, Cameron SE, Parra JL, Jones PG, Jarvis A (2005) Very high resolution interpolated climate surfaces for global land areas. Int J Climatol 25:1965-1978

Hong Y, Nix HA, Hutchinson MF, Booth TH (2005) Spatial interpolation of monthly mean climate data for China. Int J Climatol 25:1369-1379

Jeffrey SJ, Carter JO, Moodie KB, Beswick AR (2001) Using spatial interpolation to construct a comprehensive archive of Australian climate data. Environmental modelling \& Software 16:309-330

Jones RG, Noguer M, Hassell DC, Hudson D, Wilson SS, Jenkins GJ, Mitchell JFB (2004) Generating High Resolution Climate Change Scenarios using PRECIS. Met Office Hadley Centre, Exeter UK

Lemmen DS, Warren FJ, Lacroix J, Bush E (2008) From Impacts to adaptation: Canada in a Changing Climate 2007. Government of Canada, Ottawa, ON
Naoum S, Tsanis IK (2004) Ranking spatial interpolation techniques using a GIS-based DSS. Global Nest 6:1-20

Van der Heijden S, Haberlandt U (2010) Influence of spatial interpolation methods for climate variables on the simulation of discharge and nitrate fate with SWAT. Adv Geosci 27:91-98

Wu T, Li Y (2013) Spatial interpolation of temperature in the United States using residual kriging. Appl Geogr 44:112-120

doi:10.1186/2193-2697-3-8

Cite this article as: Zhou and Zhang: Application of GIS in downscaling regional climate model results over the province of Ontario.

Environmental Systems Research 2014 3:8.

\section{Submit your manuscript to a SpringerOpen ${ }^{\odot}$ journal and benefit from:}

- Convenient online submission

- Rigorous peer review

- Immediate publication on acceptance

- Open access: articles freely available online

- High visibility within the field

- Retaining the copyright to your article

Submit your next manuscript at $>$ springeropen.com 